\title{
Comparison of Spray and Point Inoculation To Assess Resistance to Fusarium Head Blight in a Multienvironment Wheat Trial
}

\author{
T. Miedaner, M. Moldovan, and M. Ittu
}

First author: University of Hohenheim (720), State Plant Breeding Institute, D-70593 Stuttgart, Germany; second author: Agricultural Research Station, Str. Agriculturii 27, 3350 Turda, Romania; and third author: Research Institute for Cereals and Industrial Crops, Jud. Călărasi, 8264 Fundulea, Romania.

Accepted for publication 18 March 2003.

\begin{abstract}
Miedaner, T., Moldovan, M., and Ittu, M. 2003. Comparison of spray and point inoculation to assess resistance to Fusarium head blight in a multienvironment wheat trial. Phytopathology 93:1068-1072.

Fusarium head blight (FHB, scab), caused by Fusarium graminearum or $F$. culmorum, results in yield and quality reductions and accumulation of mycotoxins. Two inoculation methods are commonly used. Spraying a spore suspension on the head (spray inoculation) will detect resistance to initial infection (type I) and to disease spread within the spike (type II). Injecting a spore suspension into individual florets (point inoculation) will detect type II resistance only. To analyze the association of spray and point inoculation, 20 elite winter wheat cultivars from Romania, Germany, and Switzerland were inoculated in factorial field experiments in seven environments (location $\times$ year combinations) in Germany and

Point and spray inoculations resulted in a mean disease severity varying from 52 to $63 \%$. Significant $(P=0.01)$ genotypic variation was found within and across the environments. Genotype-environment interaction was important also. Estimates of entry-mean heritability were higher for spray than for point inoculation as assessed by percent infected spikelets $(0.81$ versus 0.77$)$ and relative head weight $(0.77$ versus 0.52$)$. Significant $(P=0.01)$ interaction was found between inoculation methods. Consequently, coefficients of phenotypic correlation between both methods were low to medium for percent infected spikelets $(0.40$, $P>0.1)$ and relative head weight $(0.52, P=0.05)$. We conclude that the application of both inoculation methods should provide additional information for selection and scientific studies. Spray inoculation, however, is less laborious for large-scale routine screening of breeding materials.
\end{abstract} Romania. Response to FHB was assessed by the percentage of visually infected spikelets and head weight relative to the noninoculated control.

Fusarium head blight (FHB), caused by Fusarium graminearum Schw. (teleomorph Gibberella zeae Schwein. Petch) or F. culmorum (W.G. Smith) Sacc., has become a major problem in humid wheat (Triticum aestivum L.) producing areas $(4,12,24)$. When susceptible wheat genotypes are infected during flowering, the infected spikelets bleach prematurely. The disease progresses up and down the head and may infect all spikelets in a head when weather conditions are favorable (19). This causes considerable yield and quality losses and accumulation of mycotoxins in the grain $(12,24)$. The more resistant a genotype is, the fewer heads per plot and spikelets per head are infected. In extremely resistant wheats only some individual spikelets are affected, even when a highly aggressive isolate is successfully inoculated. Although cultural practices will also reduce disease incidence (12), growing of resistant cultivars is the most practicable and effective means of controlling FHB. Several types of genetic resistance to FHB in common wheat have been described: (i) resistance to initial infection and/or penetration, (ii) resistance to spread of disease within the spike, both first described by Schroeder and Christensen (21), (iii) resistance to kernel infection (13), and (iv) nonaccumulation or ability to degrade mycotoxins (17). Additional involvement of passive and/or tolerance components of resistance to FHB in wheat have been reported by Mesterházy (13). A relationship between the various FHB traits and resistance mechanisms was postulated (2). Type I and II resistance have been analyzed by several researchers (20) and do not seem to be genetically related. Using seven wheat genotypes tested in the greenhouse, Schroeder and

Corresponding author: T. Miedaner; E-mail address: miedaner@uni-hohenheim.de

Publication no. P-2003-0618-01R

(C) 2003 The American Phytopathological Society
Additional keyword: Triticum aestivum.

Christensen (21) found wheat lines with both types I and II present and others having only one of the two types. They distinguished the resistance components by different inoculation methods. Injecting conidia in a single floret per head (point inoculation) and observing the disease spread clearly allows the assessment of type II resistance. By spraying wheat heads with conidial suspensions and estimating disease severity, however, both type I and II resistances can be measured. For developing resistant germ plasm, artificial inoculation is indispensable to ensure a minimum disease severity every year, to optimize genotypic host differentiation, and to reduce the influence of morphological characters like awning or dwarfing (13). Type II resistance is often assessed, based on point inoculation, in greenhouse evaluations, whereas spray inoculations are mainly used in breeding programs to evaluate large host populations $(14,20)$; however, in Eastern Europe and China, point inoculations are also used in the field $(8,11)$. In molecular marker studies, different working groups use one or both methods of inoculation (10). To interpret these results, it is of utmost importance to know whether both inoculation methods will reveal the same ranking of genotypes across a larger number of environments. Environment and genotype-environment interaction have a strong impact in this pathosystem $(7,14,16)$. Our objectives were to test the covariation between spray and point inoculation across seven environments and compare the heritabilities for the two methods.

\section{MATERIALS AND METHODS}

Plant materials and design of experiments. Ten winter wheat breeding lines and registered cultivars from Romania, nine registered winter wheat cultivars from Germany, and one registered Swiss winter wheat cultivar were used. Genotypes were grown at 
Hohenheim $(\mathrm{HOH})$ on the "Filder plain" south of Stuttgart, Germany ( $400 \mathrm{~m}$ above sea level, $8.5^{\circ} \mathrm{C}$ mean annual temperature, $685 \mathrm{~mm}$ mean annual precipitation; geographic location: latitude $48.8^{\circ}$, longitude $9.2^{\circ}$ ) in 1998 and 2000 , at Fundulea (FUN) near Bucharest, Romania, in the Danube plain $(67 \mathrm{~m}$ above sea level, $10.4^{\circ} \mathrm{C}$ mean annual temperature, $571 \mathrm{~mm}$ mean annual precipitation; geographic location: latitude $44.4^{\circ}$, longitude $26.1^{\circ}$ ) in 1998, 1999, and 2000, and at Turda (TUR) in the Transylvanian Highlands, Romania $\left(345 \mathrm{~m}\right.$ above sea level, $8.8^{\circ} \mathrm{C}$ mean annual temperature, $960 \mathrm{~mm}$ mean annual precipitation; geographic location: latitude $46.6^{\circ}$, longitude $23.8^{\circ}$ ) in 1998 and 1999 . Genotypes were planted in four-row plots with $0.21 \mathrm{~m}$ between rows and $1.2 \mathrm{~m}$ long at a seed density of approximately $350 \mathrm{kernel} \mathrm{m}^{-2}$ in three replicates. A split-plot design was used, with main plots representing the three inoculation treatments (noninoculated, point inoculated, and spray inoculated) and subplots, with the genotypes randomized according to a complete block design.

Inoculation. A single-spore isolate of $F$. culmorum (FC 46) was used for inoculation. FC 46 is a highly aggressive deoxynivalenol (DON) producer isolated from winter wheat in the Netherlands in 1966 and was first described as IPO 39-01 (23). Preparation of inoculum was the same as previously described (15). In short, a spore suspension was incubated with autoclaved wheat grain in glass flasks for 4 weeks, and afterwards, the mycelium-wheat mixture was exposed to permanent UV for 4 to 7 days at $18^{\circ} \mathrm{C}$. The mixture was dried at room temperature and stored in a refrigerator at 2 to $6^{\circ} \mathrm{C}$ until usage. The mycelium-wheat mixture was suspended in tap water for $2 \mathrm{~h}$ at the inoculation date and filtered, and the conidia suspension was adjusted to a concentration of $5 \times$ $10^{5}$ spores per $\mathrm{ml}$ with $0.1 \%$ Tween 20 added. For spray inoculation, the suspension was applied at a rate of $100 \mathrm{ml} \mathrm{m}^{-2}$ by a portable sprayer designed to cover the whole plot with one spraying and equipped with a portable compressor to give a standardized pressure of 3 bars (=43.5 psi). For point inoculation, a $10-\mu 1$ droplet was injected by a syringe directly through the glumes in a central floret of each side of 20 arbitrarily chosen heads per plot that were marked by a colored clip. Each genotype was inoculated at its respective mid-flowering, and both methods of inoculation were always performed on the same date for a given genotype.

Resistance traits. Disease severity was assessed in the pointinoculated treatment by counting the number of visually diseased spikelets 10 and 20 days after inoculation and by relating them to the total number of spikelets of the respective head, resulting in percent infected spikelets. In the spray-inoculated treatment, disease progress was determined by rating the percentage of infected spikelets on a whole-plot basis at three successive dates. Because all assessments within each method were tightly correlated $(P=0.01)$, we show the respective terminal assessment only. For determining head weight, the 20 inoculated heads of the pointinoculated treatment and 20 arbitrarily chosen main-tiller spikes per plot of the spray-inoculated and noninoculated treatments, respectively, were harvested by hand at full ripening. All samples were threshed with a minimum of forced air in a single-head thresher to save highly infected, shriveled and degenerated kernels and weighed. Relative head weights for the two inoculated treatments were calculated by dividing head weight from a plot by the mean of the respective genotype in the noninoculated treatment.

Statistical analyses. All analyses were based on plot means. Single plant observations of the point-inoculated treatment were also averaged plot-wise to reduce the within-plot error. Entry means and residuals for individual environments followed a normal distribution for all traits, and error variances were homogeneous across environments according to Bartlett's test (22). The location $\times$ year combinations were analyzed as a series of random environments, using entry means and effective error mean squares (3). Estimates of variance components for genotypic variance $\left(\sigma_{\mathrm{g}}^{2}\right)$, genotype-environment interaction variance $\left(\sigma_{\text {ge }}^{2}\right)$, and error variance $\left(\sigma^{2}\right)$ were calculated as described (22). Broad-sense heritabil- ities $\left(h^{2}\right)$ were estimated on an entry-mean basis by the formula $h^{2}=\sigma_{\mathrm{g}}^{2} /\left(\sigma^{2} / r e+\sigma_{\mathrm{ge}}^{2} / e+\sigma_{\mathrm{g}}^{2}\right)$, where $r$ is the number of replicates and $e$ is the number of environments (5). Exact 90\% confidence intervals for heritability were calculated according to Knapp and Bridges (9). Standard errors of genotypic correlation coefficients were calculated according to Mode and Robinson (18). All statistical analyses were performed with the computer package PLABSTAT (version 2N; H. F. Utz, Institute of Plant Breeding, Seed Science, and Population Genetics, University of Hohenheim, Stuttgart, Germany). The effects of replicates, genotypes, and environments were assumed to be random variables, and the effect of the inoculation method was assumed to be fixed.

\section{RESULTS}

Disease severity was very high in three Romanian environments (FUN 1998, TUR 1998, and FUN 1999) and medium in the other environments (Table 1). Point and spray inoculation mostly resulted in similar disease severity in the individual environments, but there were exceptions. In 2000, for example, point inoculation was less severe than spray inoculation at both locations, but this was reflected in relative head weight only for $\mathrm{HOH}$. Conversely, both methods resulted in the same disease severity in FUN 98 in terms of percent infected spikelets, but head weight was much lower for point inoculation. Significant $(P=0.01)$ genotypic differentiation occurred for both inoculation methods and traits in all seven environments.

Both point and spray inoculation resulted in a similar mean disease severity across genotypes and environments (Table 2). For point and spray inoculation, three genotypes differed significantly $(P=0.05)$ for percent infected spikelets and relative head weight. The Romanian genotypes Fundulea 4, F92392 G3-3, and F 508U3-2 were significantly more susceptible when spray inoculated, as assessed by infected spikelets. In contrast, Kimon, F 143 T3-103, and F 91063 G3-21 had a lower relative head weight when treated by point inoculation. The Romanian breeding line Fundulea 201-R was among the most resistant genotypes for both methods and traits. Significant genotypic variation was detected in the combined analysis across environments in all instances (Table 3). The effects of environment and genotype-environment interaction were, however, considerably larger. The influence of replicates within environments was low, although significant. For point inoculation, the error variances of infected spikelets and relative head weight were 4 and 1.5 times larger than for spray inoculation, respectively. Accordingly, point inoculation had a lower heritability for both traits. Comparing the traits, the percentage of infected spikelets had a higher heritability than the relative head weight for both inoculation methods. Calculated across both inoculation methods, genotypic variances were significant too (Table 4). Interaction variances between genotypes and inoculation methods were also significant, amounting to two-thirds and one-third of the

TABLE 1. Mean and significance of genotypic variation for percent infected spikelets and relative head weight after point and spray inoculation, respectively, with Fusarium culmorum in seven environments averaged across 20 wheat genotypes ${ }^{\mathrm{a}}$

\begin{tabular}{lccccc}
\hline & \multicolumn{2}{c}{ Infected spikelets $(\%)$} & & \multicolumn{2}{c}{ Relative head wt. $(\%)$} \\
\cline { 2 - 3 } \cline { 5 - 6 } Environment $^{\mathrm{b}}$ & Point & Spray & & Point & Spray \\
\hline FUN 1998 & $66.4^{* *}$ & $65.0^{* *}$ & & $37.8^{* *}$ & $52.8^{* *}$ \\
TUR 1998 & $70.5^{* *}$ & $70.8^{* *}$ & & $65.6^{* *}$ & $58.5^{* *}$ \\
HOH 1998 & $37.8^{* *}$ & $33.0^{* *}$ & & $75.5^{* *}$ & $89.8^{* *}$ \\
FUN 1999 & $86.3^{* *}$ & $77.8^{* *}$ & & $45.0^{* *}$ & $48.9^{* *}$ \\
TUR 1999 & $63.6^{* *}$ & $53.0^{* *}$ & & $66.1^{* *}$ & $44.0^{* *}$ \\
FUN 2000 & $18.0^{* *}$ & $42.6^{* *}$ & & $68.7^{* *}$ & $78.1^{* *}$ \\
HOH 2000 & $19.5^{* *}$ & $65.2^{* *}$ & & $49.7^{* *}$ & $69.7 * *$ \\
\hline
\end{tabular}

a ** Indicates significant at $P=0.01$ ( $F$ test $)$.

${ }^{\mathrm{b}}$ FUN = Fundulea, Romania; TUR = Turda, Romania; $\mathrm{HOH}=$ Hohenheim, Germany. 
genotypic variance for infected spikelets and relative head weight, respectively. The most important source of variance was the interaction between inoculation method and environment, reflecting the different disease severities achieved with the two methods in some environments. Genotype-environment interaction variance surpassed genotypic variance for both traits. Also, the interaction between genotype, inoculation method, and environment was highly important. Phenotypic and genotypic correlations between the two traits were high and significant when using either inoculation method (Table 5). No significant relationship existed between point and spray inoculation assessed by infected spikelets, and there was only a weak relationship for relative head weight. The graphs in Figure 1 show the three genotypes deviating significantly from mean regression (arrows).

\section{DISCUSSION}

Mean disease severity was rather high for both inoculation methods. The winter wheat genotypes used represent the full range

TABLE 2. Mean percent infected spikelets and relative head weight after point and spray inoculation, respectively, of 20 wheat genotypes with Fusarium culmorum across seven environments ${ }^{\mathrm{a}}$

\begin{tabular}{|c|c|c|c|c|c|}
\hline \multirow[b]{2}{*}{ Genotype } & \multirow[b]{2}{*}{ Origin $^{b}$} & \multicolumn{2}{|c|}{$\begin{array}{c}\text { Infected } \\
\text { spikelets (\%) }\end{array}$} & \multicolumn{2}{|c|}{$\begin{array}{c}\text { Relative head } \\
\text { weight }(\%)\end{array}$} \\
\hline & & Point & Spray & Point & Spray \\
\hline Greif & GER & 76.7 & 63.4 & 47.5 & 59.3 \\
\hline Kimon & GER & 60.3 & 48.1 & $\underline{52.1}$ & $\underline{67.9}$ \\
\hline Fundulea 4 & ROM & $\underline{56.8}$ & 76.2 & $\overline{48.9}$ & $\overline{50.4}$ \\
\hline Arina & $\mathrm{CH}$ & 56.4 & 46.2 & 57.2 & 66.3 \\
\hline Orestis & GER & 55.4 & 67.4 & 51.3 & 51.1 \\
\hline F143 T3-103 & ROM & 55.4 & 55.6 & $\underline{50.6}$ & $\underline{67.3}$ \\
\hline Bussard & GER & 54.5 & 66.7 & 56.3 & 63.5 \\
\hline Kontrast & GER & 54.2 & 72.2 & 50.3 & 50.3 \\
\hline Bold & GER & 53.1 & 62.7 & 54.5 & 59.4 \\
\hline F-29 & ROM & 52.9 & 61.3 & 57.3 & 67.3 \\
\hline F 569U1-1 & ROM & 52.6 & 58.3 & 55.8 & 60.2 \\
\hline Ronos & GER & 52.0 & 65.3 & 65.2 & 60.3 \\
\hline F 92392 G3-3 & ROM & $\underline{50.6}$ & 73.3 & 52.7 & 59.6 \\
\hline Atlantis & GER & $\overline{49.2}$ & $\overline{48.7}$ & 66.2 & 69.6 \\
\hline F 91063 G3-21 & ROM & 48.6 & 49.2 & $\underline{59.5}$ & $\underline{74.3}$ \\
\hline Piko & GER & 48.2 & 47.1 & 59.0 & $\overline{71.1}$ \\
\hline F 508 U3-2 & ROM & $\underline{48.0}$ & $\underline{69.0}$ & 63.8 & 56.9 \\
\hline Turda-95 & ROM & 44.8 & 51.2 & 71.3 & 67.4 \\
\hline F 249T2-10U & ROM & 40.5 & 44.4 & 64.9 & 70.4 \\
\hline Fundulea 201-R & ROM & 28.2 & 40.0 & 75.0 & 68.3 \\
\hline Mean & & 51.9 & 58.3 & 58.0 & 63.1 \\
\hline $\mathrm{LSD}_{5 \% \text { Genotype }}{ }^{\mathrm{c}}$ & & 12.2 & 13.3 & 14.8 & 9.7 \\
\hline $\mathrm{LSD}_{5 \% \text { Difference }}{ }^{\mathrm{d}}$ & & & 19.0 & & 13.9 \\
\hline
\end{tabular}

a Significant $(P=0.05)$ differences between treatments are underlined.

b $\mathrm{CH}=$ Switzerland, GER = Germany, ROM = Romania.

c From the analysis of variance shown in Table 3.

${ }^{\mathrm{d}}$ From the analysis of variance shown in Table 4 .

TABLE 3. Components of variance and estimates of entry-mean heritability of percent infected spikelets and relative head weight after point and spray inoculation, respectively, with Fusarium culmorum across 20 wheat genotypes in seven environments ${ }^{\mathrm{a}}$

\begin{tabular}{|c|c|c|c|c|c|}
\hline \multirow[b]{2}{*}{ Parameter } & \multirow[b]{2}{*}{ df } & \multicolumn{2}{|c|}{ Infected spikelets (\%) } & \multicolumn{2}{|c|}{ Relative head wt. (\%) } \\
\hline & & Point & Spray & Point & Spray \\
\hline \multicolumn{6}{|l|}{ Components of variance } \\
\hline Environment (E) & 6 & $691.94 * *$ & $243.07 * *$ & $183.11 * *$ & $275.38 * *$ \\
\hline Replicates within E & 14 & $3.55 *$ & $2.25 * *$ & $5.92 * *$ & $8.52 * *$ \\
\hline Genotype (G) & 19 & $63.17 * *$ & $95.94 * *$ & $30.38 * *$ & $38.79 * *$ \\
\hline $\mathrm{G} \times \mathrm{E}$ & 114 & $92.66 * *$ & $146.86 * *$ & $170.61 * *$ & $65.41 * *$ \\
\hline Error & 266 & 121.44 & 31.80 & 72.98 & 54.11 \\
\hline Heritability $\left(h^{2}\right)$ & & 0.77 & 0.81 & 0.52 & 0.77 \\
\hline $90 \% \mathrm{CI}$ on $h^{2 \mathrm{~b}}$ & & $0.48-0.88$ & $0.58-0.90$ & $0.0-0.74$ & $0.48-0.87$ \\
\hline
\end{tabular}

a * And $* *$ indicate significant at $P=0.05$ and 0.01 , respectively $(F$ test $)$.

${ }^{\mathrm{b}}$ Confidence interval according to Knapp and Bridges (9). of resistance currently available in Europe in advanced breeding materials. The cvs. Piko, Bussard, and Arina, which are among the most resistant of the released German and Swiss cultivars (16), were moderately resistant only. This might reflect genotype-environment interaction, because five Romanian but only two German environments were tested, and the climatic differences were rather large. The Romanian genotype Fundulea 201-R was previously shown to be highly resistant (8), and this was confirmed by our study.

Genotypic differentiation occurred for point and spray inoculation; however, several genotypes reacted differently to the two methods, resulting in a large interaction variance. Five of the six genotypes reacting differently were of Romanian origin. Lack of correlation between point and spray inoculation has also been reported for Canadian spring wheat (6). According to Schroeder and Christensen (21), genotypes that are resistant to spray but susceptible to point inoculation should have type I resistance, whereas genotypes susceptible to spray but resistant to point inoculation should possess type II resistance. However, spray inoculation covers both types I and II resistances and this classification is, therefore, not as clear. Because no complete resistance is known with either type, a type I resistance can only be found if there is also some level of type II resistance present (20). Genotypes showing interaction between point and spray inoculation reacted in opposite directions depending on the resistance trait analyzed. Three genotypes (Fundulea 4, F 92392 G3-3, and F 508 U3-2) had a significantly higher percentage of infected spikelets when spray inoculated, but three different genotypes (Kimon, F 143 T3-103, and F 91063 G3-21) had a lower reduction in head weight across seven environments (Table 2). Concerning resistance traits, these genotypes seem to be exceptions rather than the rule because a tight genotypic correlation was found between both resistance traits within each inoculation method across the whole set of genotypes (Table 5) which is consistent with the literature $(13,14)$. An explanation would be the occurrence of type III resistance (13) in some genotypes tested. Such genotypes develop extensive head symptoms, but still produce sound kernels and, thus, a rather high relative head weight.

TABLE 4. Components of variance of percent infected spikelets and relative head weight after point and spray inoculation with Fusarium culmorum across 20 cultivars in seven environments ${ }^{\mathrm{a}}$

\begin{tabular}{lrcc}
\hline Source of variance & df & $\begin{array}{c}\text { Infected } \\
\text { spikelets }\end{array}$ & $\begin{array}{c}\text { Relative head } \\
\text { weight }\end{array}$ \\
\hline Inoculation method $(\mathrm{I})^{\mathrm{b}}$ & 1 & $-^{\mathrm{c}}$ & $\mathbf{- c}^{\mathrm{c}}$ \\
Genotype $(\mathrm{G})$ & 19 & $61.33^{* *}$ & $29.49^{* *}$ \\
$\mathrm{G} \times \mathrm{I}$ & 19 & $40.73^{* *}$ & $10.43^{* *}$ \\
$\mathrm{I} \times$ environment $(\mathrm{E})$ & 6 & $202.96^{* *}$ & $106.73^{* *}$ \\
$\mathrm{G} \times \mathrm{E}$ & 114 & $72.74^{* *}$ & $71.07^{* *}$ \\
$\mathrm{G} \times \mathrm{I} \times \mathrm{E}$ & 114 & $93.86^{* *}$ & $93.29^{* *}$ \\
Error & 544 & 78.12 & 64.19 \\
\hline
\end{tabular}

a ** Indicates significant at $P=0.01(F$ test $)$.

b Fixed factor.

c Negative variance component.

TABLE 5. Coefficients of phenotypic $\left(r_{\text {phen }}\right)$ and genotypic $\left(r_{\text {gen }}\right)$ correlation between resistance traits and inoculation methods after point and spray inoculation, respectively, with Fusarium culmorum across 20 wheat genotypes in seven environments

\begin{tabular}{lcc}
\hline Correlated traits & $r_{\text {phen }}{ }^{\mathrm{a}}$ & $r_{\mathrm{gen}}{ }^{\mathrm{b}}$ \\
\hline Infected spikelets vs. relative head weight & & \\
$\quad$ Point inoculation & $-0.73^{* *}$ & $-0.96++$ \\
$\quad$ Spray inoculation & $-0.85^{* *}$ & $-0.97++$ \\
Point vs. spray inoculation & & \\
$\quad$ Infected spikelets & 0.40 & $0.44+$ \\
$\quad$ Relative head weight & $0.52^{*}$ & $0.71++$ \\
\hline
\end{tabular}

a * And ** indicate significant at $P=0.05$ and 0.01 , respectively.

$\mathrm{b}+$ And ++ indicate that estimate is significantly greater than once and twice its standard error, respectively. 
The study clearly shows that not only the FHB resistance itself $(13,14,16,20)$, but also individual resistance components are quantitatively inherited. No genotype in this study had complete type I or type II resistance. Thus, it might be reasonable to argue that most genotypes combine quantitatively different levels of both type I and II resistances and in some cases the difference between both types just reached statistical significance (Table 2). The covariation between the two methods was reduced mainly by medium to highly susceptible genotypes, while the most resistant breeding line, Fundulea 201-R, could be identified by both methods. The poor correlation between the two methods supports the assumption that types I and II resistances might be governed by different loci and measure different resistance reactions as already suggested by Schroeder and Christensen (21). Indeed, quantitative trait loci (QTL) mapping seems to substantiate these conclusions. Yang et al. (25) found three QTLs for FHB resistance in a population of 174 spring wheat lines segregating for a Sumai 3-derived resistance. Two major QTLs on chromosomes 3BS and 6BS were significantly associated with both point and spray inoculation. Another QTL on chromosome 5AS was significantly associated with spray inoculation only. It could, therefore, be worthwhile for breeders to stack these resistance genes (2) to make rapid progress in types I and II resistance and achieve the highest resistance level possible. For identification of highly resistant parents and recombinants of types I and II resistances in segregating populations, it is necessary to apply both methods. Molecular markers might facilitate and accelerate this process $(10,25)$.

For routine screening of FHB resistance in large breeding populations, the faster, cheaper, and more reliable inoculation method is preferable. Summarizing our data, spray inoculation apparently is advantageous over point inoculation. It is based on whole-plot inoculation, which shows more similarity to natural disease occurrence and requires less time and labor for inoculation and rating. Even more importantly, spray inoculation showed higher heritabilities for both traits, i.e., the expected selection gain will be higher than for point inoculation. For analyzing resistance to mycotoxin accumulation, spray inoculation might also be preferable because multiple infection sites were observed to be generally related to higher DON contents (1). In view of the high genotypeenvironment interaction found for the two resistance components, multienvironmental tests are indispensable to reliably rank the genotypes. Genotype-environment interaction tended to be smaller, although still significant $(P=0.01)$, when the environments were situated within the same country $(14,16)$. However, sources of resistance to FHB are distributed worldwide $(13,20)$ and resistance tests across wide ranges of environments are necessary to analyze the environmental stability of possible donors. To withstand natural infections, wheat cultivars need a high expression of both resistance components to be competitive. Spore flow arrives most likely by splash dispersal, reaching the head from outside (19). Type I resistance can reduce infection efficiency. Because it is not fully efficient, some Fusarium propagules will be successful in infecting the head and then type II resistance should be activated to limit disease progress, and consequently, limit yield losses and mycotoxin content in the grain.

\section{LITERATURE CITED}

1. Bai, G.-H., Desjardins, A. E., and Plattner, R. D. 2000. Deoxynivalenolnonproducing Fusarium graminearum causes initial infection but does not cause disease spread in wheat spikes. Mycopathologia 153:91-98.

2. Ban, T. 2000. Analysis of quantitative trait loci associated with resistance to Fusarium head blight caused by Fusarium graminearum Schwabe and of resistance mechanisms in wheat (Triticum aestivum L.). Breed. Sci. 50:131-137.

3. Cochran, W. G., and Cox, G. M. 1957. Experimental Designs. 2nd ed. John Wiley \& Sons, New York.

4. Dubin, H. J., Gilchrist, L., Reeves, J., and McNab, A. (eds.) 1997. Fusarium head scab: Global status and future prospects. CIMMYT, Mexico, D.F.

5. Fehr, W. R. 1987. Principles of Cultivar Development. Vol. 1: Theory and Technique. Macmillan Publishing, New York.

6. Gilbert, J., Fedak, G., Procunier, J. D., Aung, T., and Tekauz, A. 1997. Strategies for breeding for resistance to Fusarium head blight in Canadian spring wheat. Pages 47-51 in: Fusarium Head Scab: Global Status and Future Prospects. H. J. Dubin, L. Gilchrist, J. Reeves, and A. McNab, eds. CIMMYT, Mexico, D.F.

7. Groth, J. V., Ozmon, E. A., and Busch, R. H. 1999. Repeatability and relationship of incidence and severity measures of scab of wheat caused by Fusarium graminearum in inoculated nurseries. Plant Dis. 83:10331038.

8. Ittu, M., Sãulescu, N. N., and Ittu, G. 1997. Breeding for resistance to Fusarium head blight in Romania. Pages 87-92 in: Wheat: Prospects for Global Improvement. H. J. Braun, F. Altay, W. E. Kronstad, S. P. S.

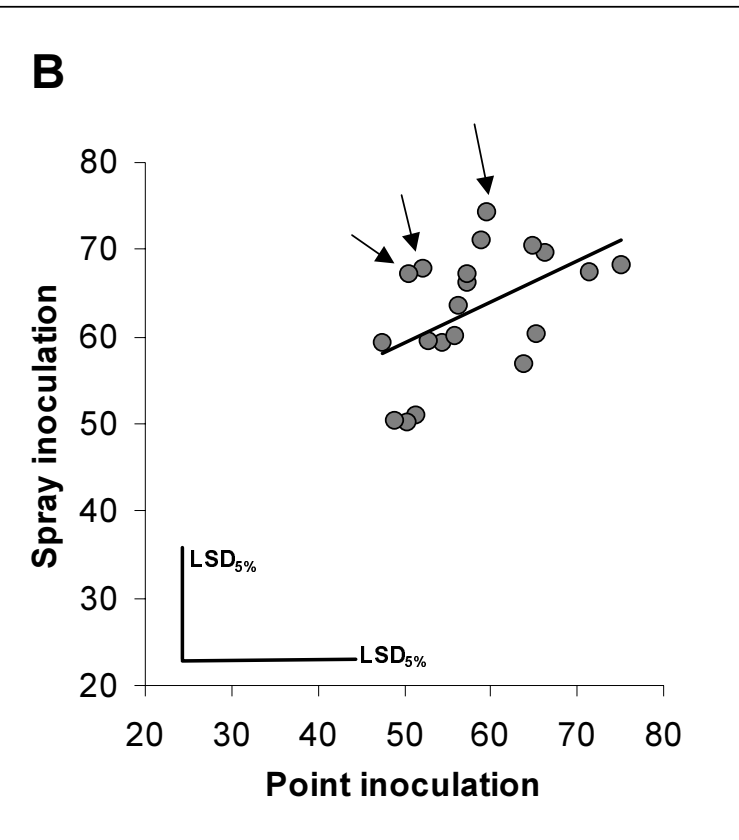

Fig. 1. Association between point and spray inoculation after infection with Fusarium culmorum for A, percent infected spikelets (\%) and B, relative head weight $(\%)$ of 20 wheat cultivars across seven environments. The line marks the regression; the arrows indicate the genotypes that deviated significantly $(P=$ 0.05 ) from the mean regression. $\mathrm{LSD}_{5 \%}$ indicates the least significant difference at $P=0.05$. 
Beniwal, and A. McNab, eds. Kluwer Academic Publishers, Dordrecht, the Netherlands.

9. Knapp, S. J., and Bridges, W. C. 1987. Confidence interval estimators for heritability for several mating and experiment designs. Theor. Appl. Genet. 73:759-763.

10. Kolb, F. L., Bai, G.-H., Muehlbauer, G. H., Anderson, J. A., Smith, K. P., and Fedak, G. 2001. Host plant resistance genes for Fusarium head blight: Mapping and manipulation with molecular markers. Crop Sci. 41:611-619.

11. Liu, Z. Z. 1984. Recent advances in research on wheat scab in China. Pages 174-181 in: Wheats for More Tropical Environments. CIMMYT, Mexico, D.F.

12. McMullen, M., Jones, R., and Gallenberg, D. 1997. Scab of wheat and barley: A re-emerging disease of devastating impact. Plant Dis. 81:1340-1348.

13. Mesterházy, Á. 1995. Types and components of resistance to Fusarium head blight of wheat. Plant Breed. 114:337-386.

14. Miedaner, T. 1997. Breeding wheat and rye for resistance to Fusarium diseases. Plant Breed. 116:201-230.

15. Miedaner, T., Gang, G., and Geiger, H. H. 1996. Quantitative-genetic basis of aggressiveness of 42 isolates of Fusarium culmorum for winter rye head blight. Plant Dis. 80:500-504.

16. Miedaner, T., Reinbrecht, C., Lauber, U., Schollenberger, M., and Geiger, H. H. 2001. Effects of genotype and genotype $\times$ environment interaction on deoxynivalenol accumulation and resistance to Fusarium head blight in rye, triticale, and wheat. Plant Breed. 120:97-105.
17. Miller, J. D., Young, J. C., and Sampson, D. R. 1985. Deoxynivalenol and Fusarium head blight resistance in spring cereals. J. Phytopathol. 113:359-367.

18. Mode, C. J., and Robinson, H. F. 1959. Pleiotropism and the genetic variance and covariance. Biometrics 15:518-537.

19. Parry, D. W., Jenkinson, P., and McLeod, L. 1995. Fusarium ear blight (scab) in small grain cereals-A review. Plant Pathol. 44:207-238.

20. Rudd, J. C., Horsley, R. D., McKendry, A. L., and Elias, E. M. 2001. Host plant resistance genes for Fusarium head blight: Sources, mechanisms, and utility in conventional breeding systems. Crop Sci. 41:620627.

21. Schroeder, H. W., and Christensen, J. J. 1963. Factors affecting resistance of wheat to scab caused by Gibberella zeae. Phytopathology 53:831-838.

22. Snedecor, G. W., and Cochran, W. G. 1989. Statistical Methods, 8th ed. Iowa State University, Ames, IA.

23. Snijders, C. H. A., and Perkowski, J. 1990. Effects of head blight caused by Fusarium culmorum on toxin content and weight of wheat kernels. Phytopathology 80:566-570.

24. Windels, C. E. 2000. Economic and social impacts of Fusarium head blight: Changing farms and rural communities in the Northern Great Plains. Phytopathology 90:17-21.

25. Yang, Z., Gilbert, J., and Fedak, G. 2002. New microsatellite markers for type I and type II resistance to Fusarium head blight in spring wheat. J. Appl. Genet. 43A:359-362. 